\title{
Curcumin Improves Chronic Pain Induced Depression Through Regulating Serum Metabolomics in a Rat Model of Trigeminal Neuralgia
}

This article was published in the following Dove Press journal:

Journal of Pain Research

\author{
Li Zhang $\mathbb{D}^{1, *}$ \\ Zhijie $\mathrm{Ma}^{2}$ \\ Zhe $\mathrm{Wu}^{3}$ \\ Mu Jin' \\ Lixin An ${ }^{\prime}$ \\ Fushan Xue $\mathbb{D}^{1}{ }^{1, *}$ \\ 'Department of Anesthesiology, Beijing \\ Friendship Hospital, Capital Medical \\ University, Beijing 100050, People's \\ Republic of China; ${ }^{2}$ Department of \\ Pharmacy, Beijing Friendship Hospital, \\ Capital Medical University, Beijing, \\ People's Republic of China; ${ }^{3}$ Department \\ of Anesthesiology, Pain Medicine \& \\ Critical Care Medicine, Aviation General \\ Hospital of China Medical University, \\ Beijing 1000I2, People's Republic of \\ China \\ *These authors contributed equally to \\ this work
}

Background: Depression is a prevalent and complex psychiatric disorder with high incidence in patients with chronic pain. The underlying pathogenesis of chronic pain-induced depression is complicated and remains largely unclear. An integrated analysis of endogenous substance-related metabolisms would help to understand the molecular mechanism of chronic pain-induced depression. Curcumin was reported to exert various health benefits, such as anti-depression, antioxidant, antineoplastic, analgesia, and anti-inflammation.

Objective: The aim of this study was to analyze the biomarkers related to depression in serum and to evaluate the anti-depression properties of curcumin in a chronic pain-induced depression model of rats.

Design: This is a randomized, controlled experiment.

Setting: This study was conducted at the Experimental Animal Center, Beijing Friendship Hospital, Capital Medical University.

Methods: Trigeminal neuralgia (TN) was produced by injecting $4 \mu \mathrm{L}, 10 \%$ cobra venom saline solution into the infraorbital nerve (ION). Curcumin was administered by gavage twice a day from post-operation day (POD) 15 to POD 42. Mechanical allodynia was assessed using von Frey filaments. Sucrose preference and forced swimming tests were performed to evaluate depression-like behaviors. The metabolomics analysis was preceded by LCMS-IT-TOF and multivariate statistical methods for sample detection and biomarker screening.

Results: Cobra venom intra-ION injection led to chronic mechanical allodynia, reduced sucrose preference, and prolonged immobility during forced swimming. Curcumin treatment alleviated chronic mechanical allodynia, regained sucrose preference, and reduced immobility time. Differential analysis identified 30 potential metabolites changed under TN condition. The integrated analyses further revealed two major metabolic changes by comparing the serums from sham operated rats, TN rats, and TN rats treated with curcumin: 1) ether lipid metabolism; and 2) glycerophospholipid metabolism, and suggested that curcumin may improve chronic pain-induced depression by regulating these two types of lipid metabolisms. Conclusion: Ether lipid and glycerophospholipid metabolism might be two of the pathways with the most potential related to chronic pain induced-depression; and curcumin could alleviate chronic pain induced-depression by modulating these two pathways. These results provide further insights into the mechanisms of chronic pain-induced depression and may help to identify potential targets for anti-depression properties of curcumin.

Keywords: curcumin, depression, chronic pain, trigeminal neuralgia, metabolomics
Correspondence: Li Zhang; Fushan Xue Department of Anesthesiology, Beijing Friendship Hospital, Capital Medical University, No. 95, Yong'an Road, Xicheng District, Beijing 100050, People's Republic of China

Email zhangli8828@163.com;

xuefushan@aliyun.com
Journal of Pain Research 2020:13 3479-3492

\section{(ce)} (c) (i) (5) 2020 Zhang et al. This work is published and licensed by Dove Medical Press Limited. The full terms of this license are available at https://www.dovepress.com/terms. work you hereby accept the Terms. Non-commercial uses of the work are permitted without any further permission from Dove Medical Press Limited, provided the work is properly attributed. For permission for commercial use of this work, please see paragraphs 4.2 and 5 of our Terms (https://www.dovepress.com/terms.php). 


\section{Introduction}

Chronic pain patients often develop into depression,, which leads to additional emotional and cognitive deficits, ${ }^{3,4}$ and has become a general medical problem. The involvements of anatomical, neurochemical, and psychological changes in depression induced by chronic pain have been intensively studied. ${ }^{5-7}$ However, the potential contributions of microcosmic metabolite changes under pathologic pain conditions to development of depression has not had enough attention yet. As a powerful approach to directly reflect the underlying biochemical activity and state of cells or tissues, metabolome reveals the entire metabolic profile by detecting over 1,000 molecules in various biological samples, such as blood, urine, saliva, and cerebrospinal fluid. ${ }^{8}$ By analyzing specific biomarkers, metabolic profiling can provide a complete insight of disease progression and the outcome of drug treatment by functional readout of the physiological state of a whole biologic system. ${ }^{9}$

Due to its antioxidant, anti-inflammatory, ${ }^{10}$ antimutagenic, antimicrobial, ${ }^{11,12}$ and anticancer properties, ${ }^{13,14}$ curcuma longa has been traditionally used in Asian countries as a medical herb. Curcumin (1,7-bis(4-hydroxy3-methoxyphenyl)-1,6-heptadiene-3,5-dione), also called diferuloylmethane, is the main natural polyphenol found in the root of turmeric (Curcuma longa) and in others Curcuma spp. [PMID:12680238], and has been shown to target multiple signaling molecules while also demonstrating activity at the cellular level, which has helped to support curcuma longa multiple health benefits. ${ }^{15}$ Curcumin has been shown to benefit pain and depression through inhibiting monoamine oxidase, modulating the level of serotonin and monoamine, inhibiting glutamate release in the prefrontal cortex, ${ }^{16-18}$ and activating MAPK/ERK-dependent BDNF expression in the amygdala region. ${ }^{19}$ However, a detailed metabolic profiling for the anti-depression effects of curcumin has not been done yet. In our previous studies, our team developed a new trigeminal neuralgia rat model induced by cobra venom injected into the infraorbital nerve (ION); the model displays persistent pain and mimics the trigeminal neuralgia onset in human patients. ${ }^{20}$

In this study, we performed a metabolomics assay using HPLC coupled with IT-TOF mass spectrometry and characterized the metabolic profiles underlying depression evoked by trigeminal neuralgia, which was induced by injecting cobra venom into the infraorbital nerve (ION), as reported in our previous study. ${ }^{20}$
Furthermore, we studied if curcumin can be used to treat depression induced by chronic pain, and its regulation to biomarkers and metabolic profiles, which were specifically changed under neuropathic conditions.

\section{Methods}

\section{Animals}

Male SD rats (200-220 g) were purchased from The Institute of Laboratory Animal Sciences, Chinese Academy of Medical Sciences \& Peking Union Medical College and used in this study. All animal procedures were approved by the Ethical Committee of Beijing Friendship Hospital, Capital Medical University (Beijing, China), and were performed in accordance with the guidelines of the International Association for the Study of Pain. Rats were housed under a 12-hour light/dark cycle and at a temperature of $22-24^{\circ} \mathrm{C}$. All rats were given food and water ad libitum. Twenty-five rats were randomly divided into three groups: sham group $(\mathrm{n}=8), \mathrm{TN}+$ vehicle group $(\mathrm{n}=8)$, and $\mathrm{TN}+$ curcumin group $(\mathrm{n}=9)$. Curcumin or vehicle was orally administered by gavage twice a day (morning and evening) from POD 15 to 42 .

\section{Drug Preparation}

Lyophilized cobra venom (Formosan cobra; Sigma, St. Louis, MO) was dissolved in $0.9 \%$ sterile saline to make $10 \%$ solution. ${ }^{20}$ Curcumin (CAS: $458-37-7$, Sigma Aldrich, purity $\geq 99.5 \%$ ) $0.3 \%$ was made in peanut oil and administered to rats in the $\mathrm{TN}+$ curcumin group $(45 \mathrm{mg} / \mathrm{kg}$, $1.5 \mathrm{~mL}$ ) based on earlier literature. ${ }^{21-23}$

\section{Blood Sample Preparation}

Orbital blood $(1.5 \mathrm{~mL})$ was collected from all rats weekly in the morning for 6 weeks except weeks 3 and 5, and centrifuged at $4,000 \times \mathrm{g}$ for 15 minutes to obtain serum, which was sterilized without hemolysis and stored at $-80^{\circ}$ C. To prepare the samples for chromatography and mass spectrometry, $200 \mu \mathrm{L}$ thawed serum was added into 600 $\mu \mathrm{L}$ methanol and mixed for 30 minutes at $4{ }^{\circ} \mathrm{C}$ followed by high-speed centrifugation at $12,000 \mathrm{rpm}$ and 10 minutes incubation at $4{ }^{\circ} \mathrm{C}$ to remove solid debris. Finally, the supernatant was filtered through a microfiltration membrane $(0.22 \mu \mathrm{m})$ to obtain the injection samples.

\section{Surgical Procedure}

Models of TN were produced according to the method described in our previous studies. ${ }^{20,22}$ Briefly, after the 
rats were anesthetized by sodium pentobarbital $(40 \mathrm{mg} / \mathrm{kg}$, i.p.), a 1-cm radical incision was made above the left superciliary arch to expose the infraorbital nerve (ION). For rats assigned to the $\mathrm{TN}$ group, $4 \mu \mathrm{L}$ cobra venom solution (10\% in saline) was injected into the ION. The incision was closed in layers. Rats in the sham group received $4 \mu \mathrm{L}$ of saline injection instead.

\section{Behavioral Testing Mechanical Sensitivity Test}

Mechanical allodynia was used to verify trigeminal neuralgia induced by cobra venom intra-ION injection. The rats were placed in a plastic chamber $(20 \mathrm{~cm} \times 25 \mathrm{~cm} \times 15 \mathrm{~cm})$ and acclimated for 15 minutes. Their mechanical withdrawal thresholds (MWT) were measured via von Frey filaments (Stoelting, Chicago, IL) and calculated through up-down method as described previously. ${ }^{20}$ Each filament was placed perpendicularly on the facial skin innervated by left ION, which is near the center of the vibrissa pad. A positive response was defined by brutally turning the head away, scratching the left side of the face, or attacking the filaments.

\section{Sucrose Preference Test}

Before the test, all rats received water and $1 \%$ sucrose solution alternately for 48 hours and fast for 24 hours. On the testing day rats were allowed to acclimate in the test room for 20 minutes and provided by a bottle of water and a bottle of $1 \%$ sucrose solution for 30 minutes, followed by another 30 minutes after swapping the two bottles. Sucrose preference was calculated by dividing the volume of consumed sucrose solution by total liquid consumption.

\section{Forced Swim Test}

In the forced swim test, immobility time was collected to evaluate depression-like behaviors. After 20 minutes acclimation in the test room rats were placed into a standard clear Porsolt chamber filled with water $\left(25^{\circ} \mathrm{C}\right.$ and $25-\mathrm{cm}$ depth) for 15 minutes, and then removed from the chamber, dried, and returned to their home cages. Twenty-four hours later, the rats were placed into the same Porsolt chamber for 5 minutes. Immobility, defined as no hind paw movement for over 1 seconds during swimming, ${ }^{24}$ was recorded and analyzed in the second session by two independent researchers who were blinded to the rats before operation, and weekly after operation for 6 weeks, except the $3^{\text {rd }}$ and $5^{\text {th }}$ week.

\section{Chromatography and Mass Spectrometry Assay of Serum Samples}

Chromatography and mass spectrometry assay were performed on a LCMS-IT-TOF system (Shimadzu, Japan). Blood serum was separated on an XBridge ${ }^{\circledR}$ C18 Column (3.5 $\mu \mathrm{m}, 2.1 \times 100 \mathrm{~mm}$, Waters). The temperature of the column was set to $40^{\circ} \mathrm{C}$. The mobile phase flow rate was $0.30 \mathrm{~mL} / \mathrm{min}$ and consisted of $0.1 \%$ formic acid in water (A) and $0.1 \%$ formic acid in acetonitrile (B). Serum sample injected $(5 \mu \mathrm{L})$ was trapped using $100 \%$ mobile phase A for 13 minutes at a flow rate of $5 \mu \mathrm{L} / \mathrm{min}$ before being placed in-line with the analytical column and subjected to the gradient profile which was set as follows: 5\% B for 3 minutes, $3-50 \%$ B for 3-5 minutes, $50 \%$ B for 3 minutes, $50-70 \%$ B for 8.0 to 10.0 minutes, $70 \%$ B for 10 minutes, $70-95 \%$ B for 20.0 to 22.0 minutes, $95 \%$ B for 3 minutes, 95-5\% B for 25.0-27.0 minutes, and 5\% B for 8 minutes. The eluent was directly introduced to the mass spectrometer. To ensure the stability and repeatability of the LCMS systems, a QC was run after every 10 samples were analyzed, and followed by a blank. The Q-Exactive mass spectrometer parameters were as follows: electrospray voltage was 1.57 $\mathrm{kV}$, Orbitrap precursor spectra (AGC $3 \times 106$ ) were collected from $100-1,000 \mathrm{~m} / \mathrm{z}$; the gas temperature was $200^{\circ} \mathrm{C}$; the gas flow was $0.8 \mathrm{~L} / \mathrm{min}$; and charge state screening was with a dynamic exclusion time of 30 seconds to discriminate against previously analyzed ions. The method was validated for selectivity, sensitivity, linearity, accuracy and precision, recovery, matrix effect, dilution integrity, and stability.

\section{Multivariate Data Analysis and Statistical Analysis of Metabolic Profiling}

Sample data were extracted using Profiling Solution software for peak detection and alignment. The full scan mode was set in the range of $100-1,000 \mathrm{~m} / \mathrm{z}$ and data collection as the initial and final retention times. Resultant data matrices was analyzed by software SIMCA-P11.0 (Umetrics, Umea, Sweden) for multivariate data analysis, such as principal component analysis (PCA) and partial least-squares discriminant analysis (PLS-DA). Prior to PCA, all data obtained from the matrix were mean centered and scaled to a Pareto variance. As an unsupervised pattern recognition approach, PCA was usually used for reducing the matrix dimension and demonstrating the intrinsic simples clustering degree. On this basis, PLSDA was used to identify the variables responsible for separation, and correlating with the variable influence on 
projection (VIP) parameter select the most significant variables contributing to discriminate metabolomics profiles between sham and $\mathrm{TN}+$ vehicle groups and between $\mathrm{TN}+$ vehicle and $\mathrm{TN}+$ curcumin groups. In a PLS-DA model, the VIP is a weighted sum of squares of the PLS weight, indicating the importance of the variable to the entire model. The variables with VIP values $\geq 1.5$ were considered as significant differences and were selected for further data analysis. Then, the compounds corresponding to the significantly changed variables between groups $(P$-value $<0.05)$ were screened as biomarkers. ${ }^{25}$ All potential biomarkers were annotated by the Human Metabolome Database (HMDB) and METLIN database. Finally, the compounds and their KEGG data numbers were imported to Metaboanalyst 3.0 for further pathway and enrichment analysis.

Behavioral data were analyzed using SPSS 19.0 Software. Statistical significance was determined $(P<0.05)$ using repeated measures two-way ANOVA.

\section{Results}

\section{Curcumin Alleviated Mechanical}

\section{Allodynia After Trigeminal Neuralgia}

Intra-ION injection of cobra venom but not saline produced a fast-onset (within 1 week) and long-lasting (over 6 weeks) mechanical allodynia, which is one of the symptoms of trigeminal neuralgia in rats. From POD 15-42, curcumin $(45 \mathrm{mg} / \mathrm{kg}$ in $1.5 \mathrm{~mL})$ was administrated twice a day by gavage (10:00 am and 18:00 pm) (Figure 1A). $\mathrm{TN}$ rats treated by curcumin exhibited a significant higher
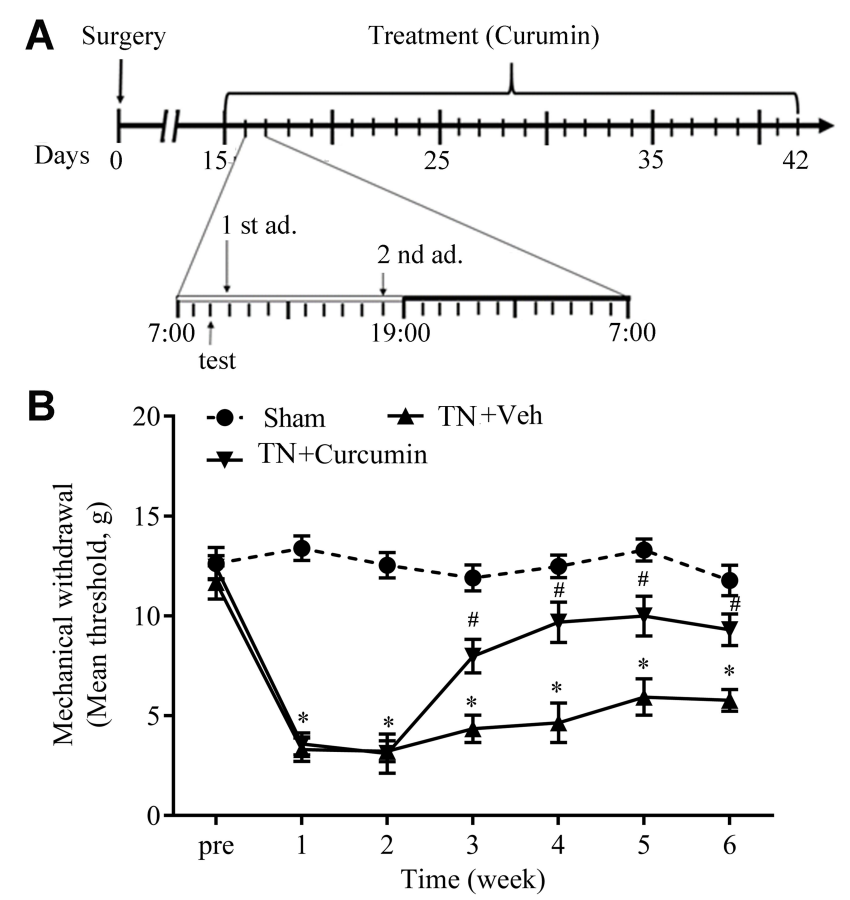

Figure I Curcumin alleviated mechanical allodynia induced by cobra venom intraION injection. (A) Schematic for the timeline of surgery (cobra venom or sterile saline injection) and curcumin administrations. (B) Mechanical allodynia induced by cobra venom intra-ION injection was attenuated by curcumin treatment. Two-way ANOVA, ${ }^{*} P<0.05$ vs the sham group, $\# P<0.05$ vs the cobra venom group, $n=8$ or 9 .

mechanical withdrawal threshold than TN rats that only received vehicle treatment $(\mathrm{F}(2,22)=17.25, \quad P<0.0001)$ (Figure 1B), suggesting long-term curcumin treatment significantly alleviated mechanical allodynia observed in intra-ION injection of cobra venom induced trigeminal neuralgia.
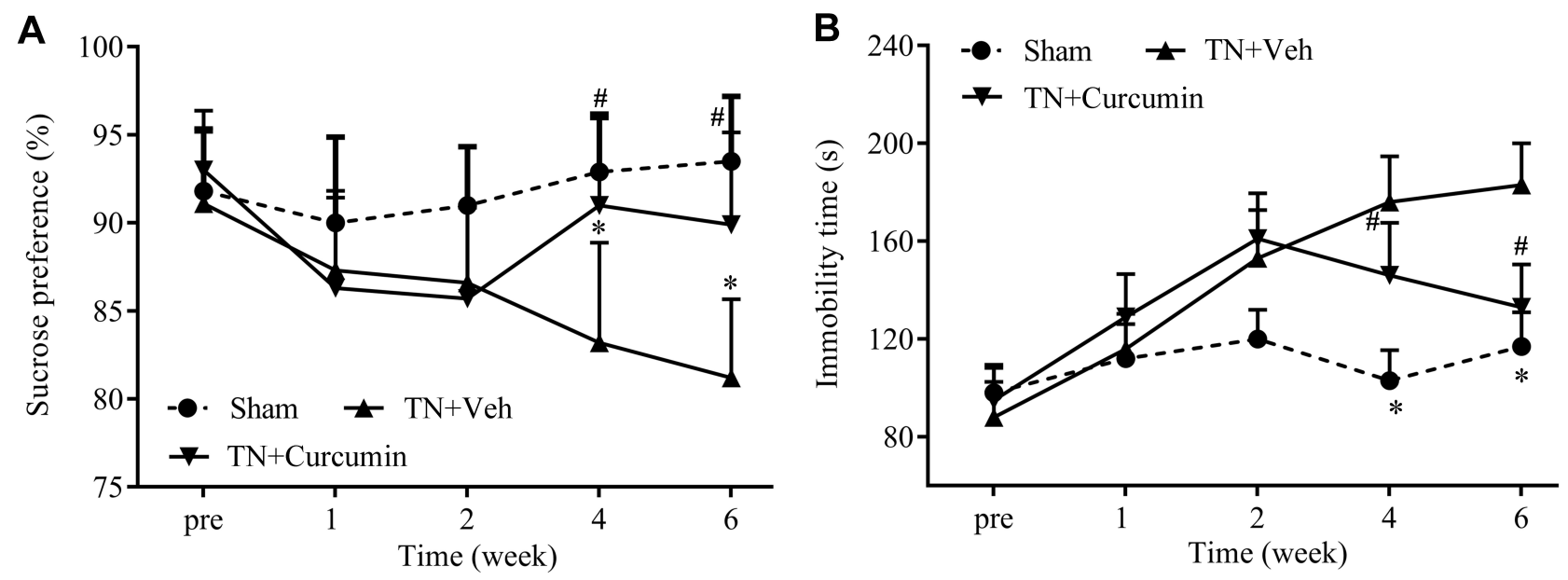

Figure 2 The effects of curcumin treatment on depression behaviors observed in rats with chronic pain. (A) Sucrose preferences were progressively reduced in rats with $\mathrm{TN}$, and recovered by curcumin treatment. (B) Immobility time during forced swimming were progressively increased in rats with TN and normalized after curcumin treatment. Two-way ANOVA, $* P<0.05$ vs the sham group, $\# P<0.05$ vs the cobra venom group, $n=8$ or 9 . 

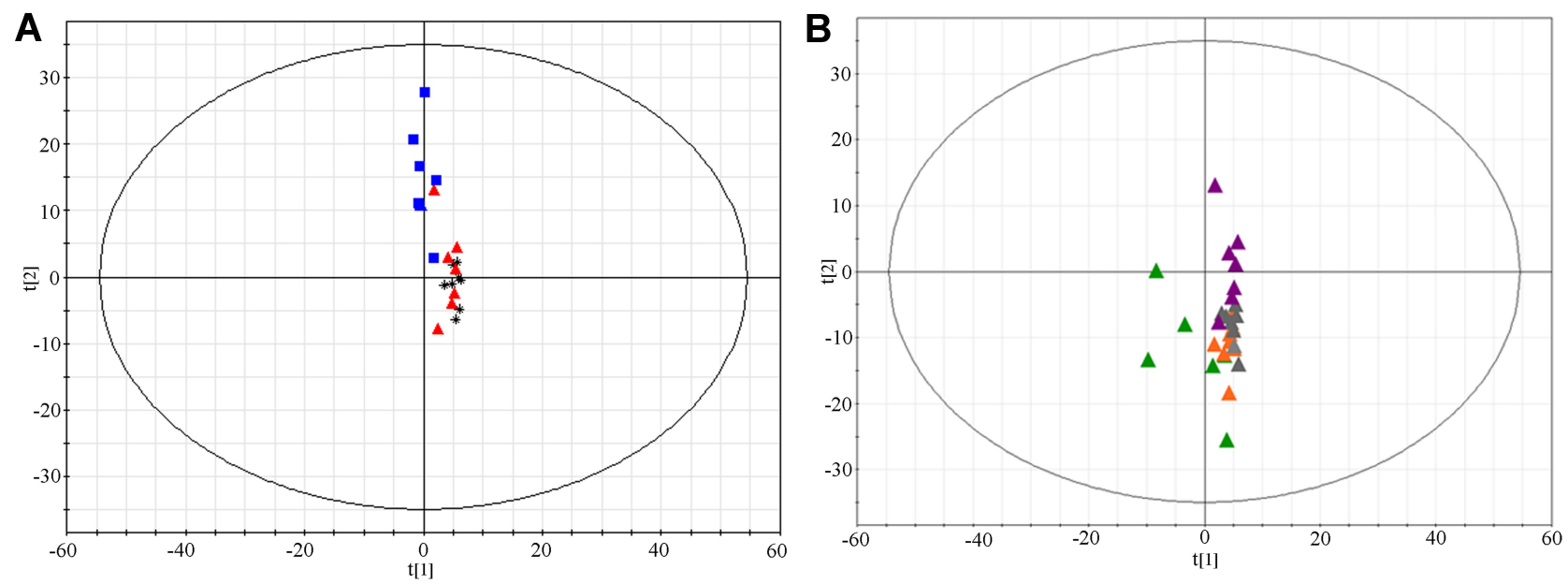

Figure 3 Score-Plot of principal component analysis for different groups of rats. (A) Score-Plot of PCA for the serum samples collected from different groups 6 weeks after surgery. Black, sham group; Red, TN+vehicle group; and Blue, TN+curcumin group. (B) Score-Plot PCA for the serum samples collected at different time points after cobra venom intra-ION injection. Green, week I; Orange, week 2; Grey, week 4; and Violet, week 6.

A
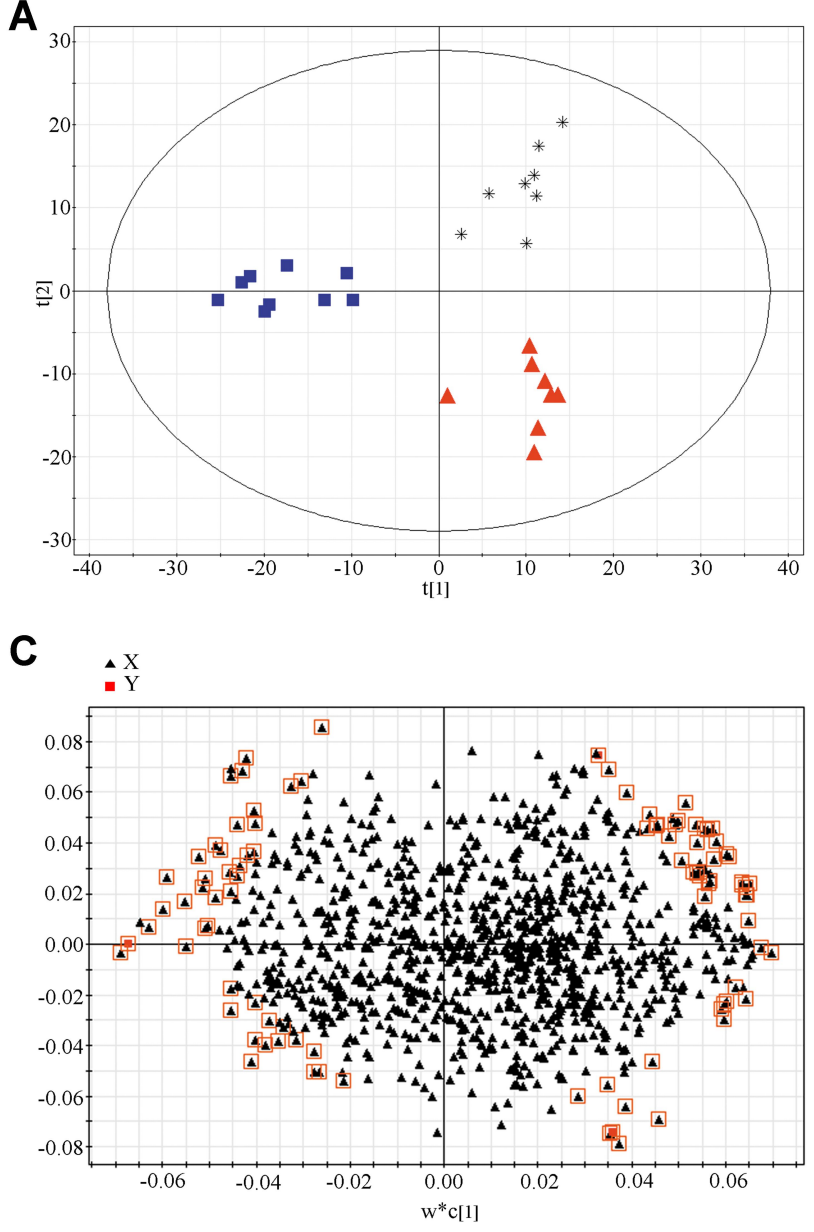

B

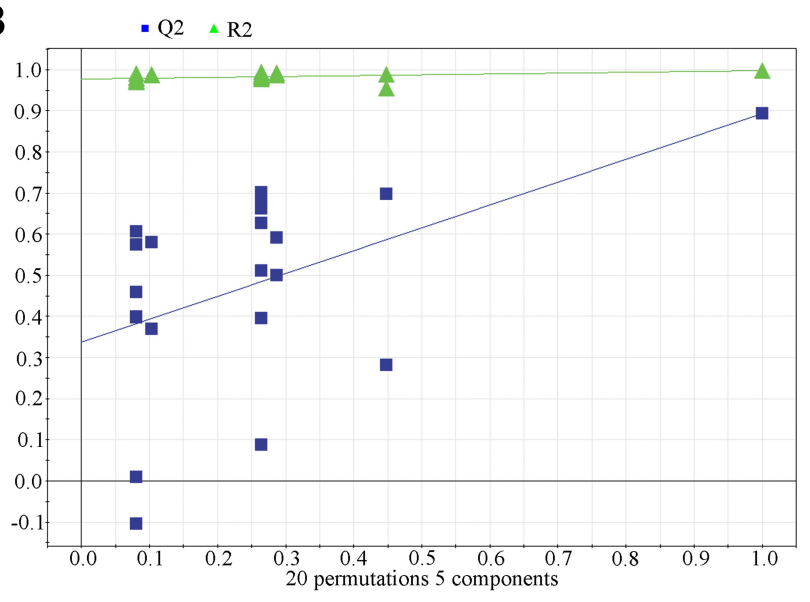

D

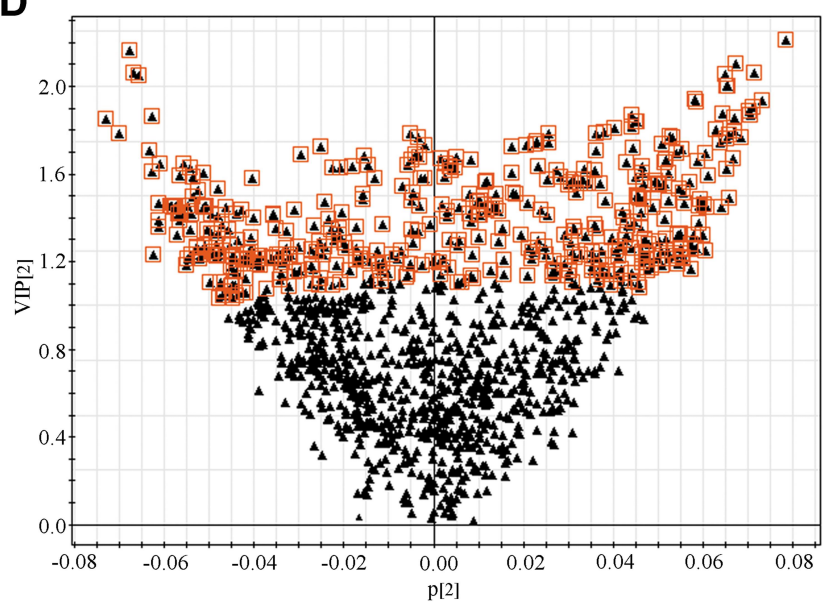

Figure 4 Partial least-squares discriminant analysis of metabolomics data in different experimental groups. (A) Score plot. Black, sham group; Red, TN group, and Blue, TN+ curcumin group. (B) 100-permutation test. (C) loading plot. (D) VIP value plot. 


\section{Curcumin Alleviated Trigeminal}

\section{Neuralgia-Induced Depression}

Forced swimming test and sucrose preference test were used to evaluate rat depression-like behaviors in this study. From POD 28, TN rats exhibited significantly less sucrose consumption and longer immobility time during swimming than rats in the sham group, indicating that $\mathrm{TN}$ induced depression-like behaviors, which on the other hand were alleviated after curcumin treatment demonstrated by regained sucrose consumption $(\mathrm{F}(2,22)=19.85$, $P<0.0001)$ and decreased immobility time during swim$\operatorname{ming}(\mathrm{F}(2,22)=66.58, P<0.0001)($ Figure $2 \mathrm{~A}-\mathrm{B})$.

\section{Changes in Serum Metabolites from Rats with Trigeminal Neuralgia with or Without Curcumin Treatments}

The PCA, as an unsupervised analysis method, was used to compare serum metabolites between sham, TN plus curcumin treatment, and TN plus vehicle groups. As shown in Figure 3A, no significant differences were detected between three groups via PCA according to their score-plot clusters. Meanwhile, no significant differences were detected between different time points (week 1, 2, 4, and 6) after cobra venom intra-ION injection (Figure 3B). To detect any potential differences between groups, which may be difficult to be found by PCA, we further used partial least-squares discriminant analysis (PLS-DA) to screen any potentially changed biomarkers. As shown in Figure 4A, the scoreplot clusters of the sham, TN, and TN plus curcumin groups were discriminated in positive models, in which the three parameters of PLS-DA R2X, R2Y, and Q2 (cum) values used for model fitting the matrix data were $0.414,0.997$, and 0.914 , indicating that the fitting model was high quality and could provide accurate predictions. The clusters in the TN group for different time points after cobra venom intra-ION injection were also discriminated in positive models (Supplementary Figure 1A), in which the R2X, R2Y, and Q2 (cum) values were $0.478,0.986$, and 0.856 , respectively. Finally, the fitting models were validated by the permutation test with 100 iterations, which compared the fitting goodness of the origin model with that of the updated model. As shown in Figure 4B and Supplementary Figure 1B, the validation plots indicated that the original models were valid. The loading plots displayed variables that positively correlated with the score plots (Figure 4C and Supplementary Figure 1C) and demonstrated in more detail several crucial variables, which were far from the center of the coordinates, and contributed the most to PLS-DA clustering. Only VIP value as shown in Figure 4D and

Table I Identified Metabolites from the Serum Collected at Different Time Points After Venom Cobra Intra-ION Injecton

\begin{tabular}{|c|c|c|c|c|c|c|}
\hline VIP & $M / Z$ & $\mathbf{R T}$ & Biomarker & {$[M+X]^{+}$} & Adduct MW & Delta (ppm) \\
\hline 2.01 & 263.1987 & 23.696 & 9-Pentadecenoic acid & 263.1981 & $\mathrm{M}+\mathrm{Na}$ & 6 \\
\hline 1.63 & 301.1398 & 29.242 & alpha-CEHC & 301.141 & $\mathrm{M}+\mathrm{Na}$ & 4 \\
\hline 1.76 & 319.1932 & 29.417 & Ubiquinone-2 & 319.1904 & $\mathrm{M}+\mathrm{H}$ & 9 \\
\hline 2.02 & 329.2457 & 28.233 & 5,8, II-Eicosatrienoic acid & 329.2451 & $\mathrm{M}+\mathrm{Na}$ & 2 \\
\hline 1.64 & 377.2642 & 26.549 & $\mathrm{G}(0: 0 / 18: 2(9 Z, \mid 2 Z) / 0: 0)$ & 377.2662 & $\mathrm{M}+\mathrm{Na}$ & 5 \\
\hline 1.54 & 379.2794 & 29.885 & $M G(0: 0 / I 8: I(I I Z) / 0: 0)$ & 379.2819 & $\mathrm{M}+\mathrm{Na}$ & 7 \\
\hline 1.61 & 413.2657 & 27.193 & 9'-Carboxy-alpha-chromanol & 413.2662 & $\mathrm{M}+\mathrm{Na}$ & I \\
\hline 1.51 & 520.3376 & 20.843 & LysoPC(18:2(9Z,12Z)) & 520.3398 & $\mathrm{M}+\mathrm{H}$ & 4 \\
\hline 1.51 & 524.3662 & 23.709 & LysoPC(0:0/I8:0) & 524.3711 & $\mathrm{M}+\mathrm{H}$ & 9 \\
\hline 1.51 & 553.3871 & 30.444 & LysoPE(22:I(I3Z)/0:0) & 553.3976 & $\mathrm{M}+\mathrm{HN} 4$ & 19 \\
\hline 1.58 & 592.34 & 23.119 & LysoPC(22:5(4Z,7Z, 10Z, I3Z, I6Z)/0:0) & 592.3374 & $\mathrm{M}+\mathrm{Na}$ & 4 \\
\hline 1.55 & 400.3399 & 23.276 & MG(0:0/20:2(IIZ,I4Z)/0:0) & 400.3421 & $\mathrm{M}+\mathrm{NH} 4$ & 6 \\
\hline 1.99 & 334.2046 & 28.061 & 2-trans,4-cis-Decadienoylcarnitine & 334.1989 & $\mathrm{M}+\mathrm{Na}$ & 17 \\
\hline 1.62 & 136.0219 & 14.858 & Lysylvaline & 263.2078 & $\mathrm{M}+\mathrm{NH} 4$ & 21 \\
\hline
\end{tabular}


Supplementary Figure 1D was above 1.5, being used to card and structurally identify potential biomarkers, and was considered as a screening standard to select metabolites and $\mid P$ (corr) $\mid$ above 0.58 .

\section{Characterizing Potential Metabolites and Metabolic Pathways}

Through PCA and combined PLS-DA we detected that serum metabolites were changed in rats with trigeminal neuralgia. Among 1,256 signals detected after trigeminal neuralgia, we selected 29 variables as candidates, which significantly contributed to the clustering and discrimination between groups, according to $\mathrm{VIP} \geq 1.5$ and $\mid P$ (corr) $\mid \geq 0.58$ (Supplementary Table 1) , to compare between weeks 1, 2, 4, and 6 after cobra venom intra-ION injection via ANOVA analyses, and found 14 candidates, which were significantly different among groups $(P<0.05)$, and recognized as candidate biomarkers according to HMDB and Metaboanalyst identification. The 14 potential biomarkers were also significantly changed along the progress of trigeminal neuralgia from week 1-6 after cobra venom injection (Table 1 and Figure 5A).

A

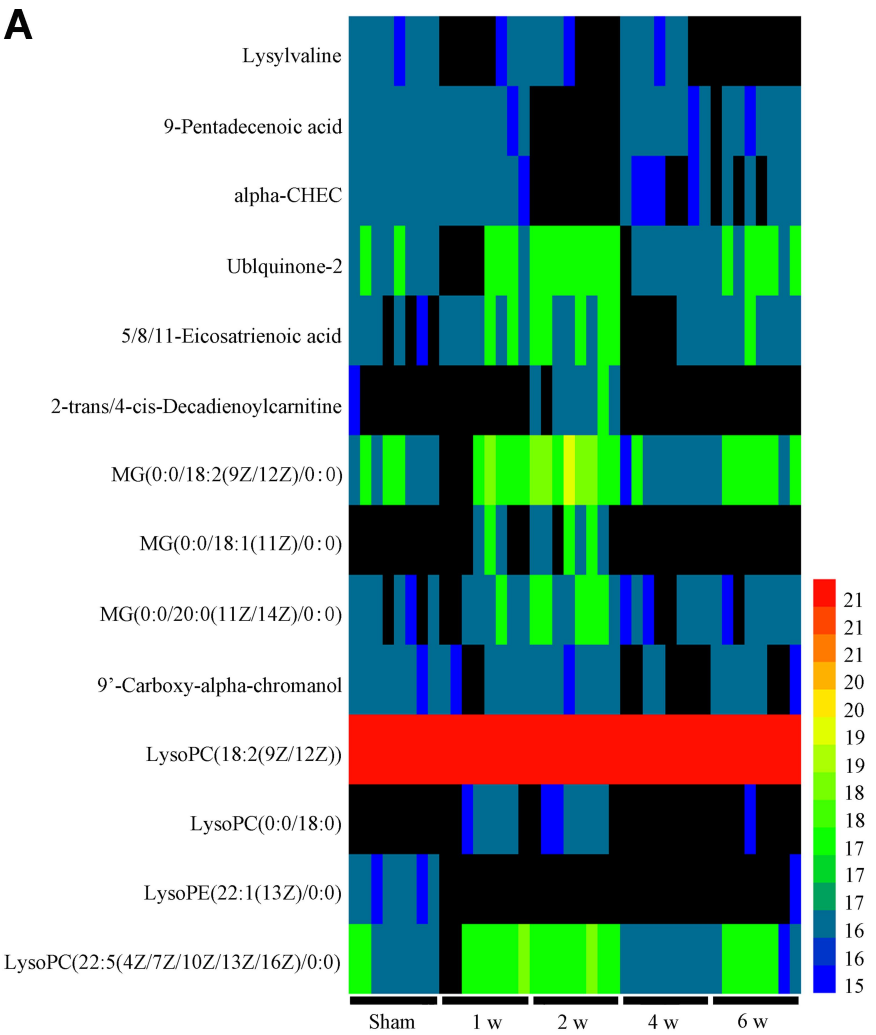

To examine if curcumin treatment changed metabolites in trigeminal neuralgia conditions, we found 29 potential biomarkers, which were significantly different between $\mathrm{TN}+$ curcumin treatment group and $\mathrm{TN}+$ vehicle group in the serum samples (Figure 5B), and are summarized in Table 2. Among them the amounts of CE (20:0), LysoPC(O-18:0), 9'Carboxy-alpha-chromanol, and LysoPC(14:1(9Z)) were much less in the $\mathrm{TN}+$ curcumin group than in the $\mathrm{TN}+$ vehicle group.

Last, we conducted metabolic pathway analysis and found that the metabolites changed along the progress of $\mathrm{TN}$ after cobra venom intra-ION injection were related to glycerophospholipid metabolism (Supplementary Figure 2). Considering that curcumin treatment can effectively relieve chronic pain and depressive-like behaviors in our TN model, we furthermore compared small molecule metabolites between the TN + curcumin treatment and $\mathrm{TN}+$ vehicle groups, and found differences in several metabolic pathways between groups such as glycerophospholipid metabolism, ether lipid metabolism, linoleic acid metabolism, alpha-linolenic acid metabolism, pentose and glucoronate interconversions, starch and sucrose metabolism, steroid biosynthesis, arachidonic acid metabolism, and tyrosine metabolism, suggesting their

B

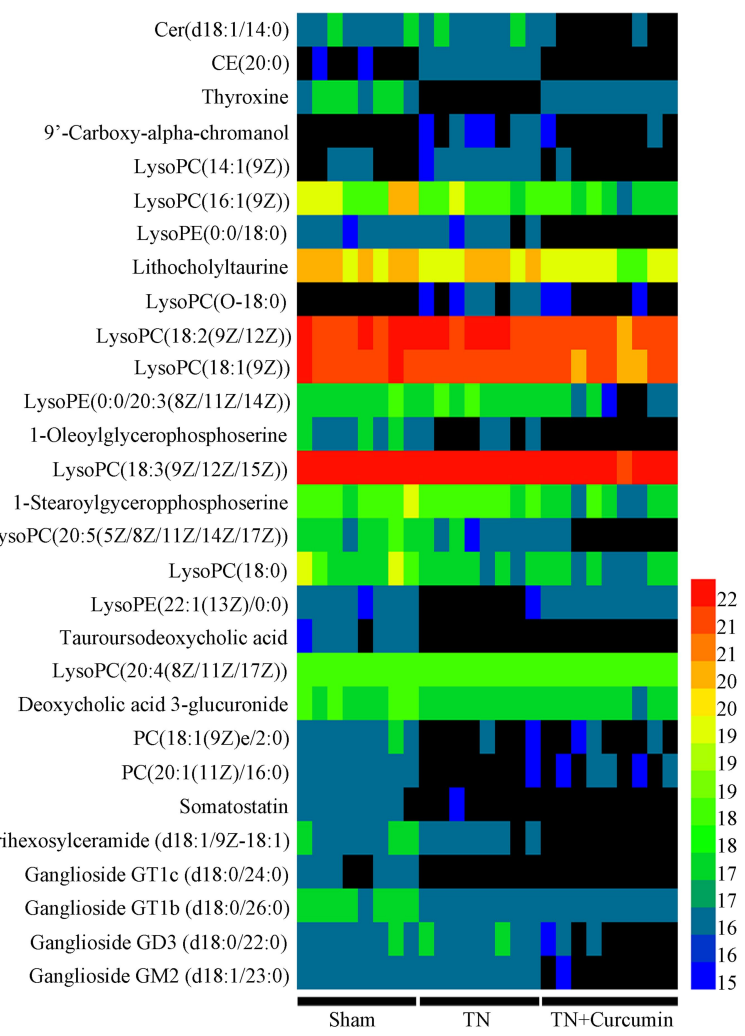

Figure $\mathbf{5}$ Serum metabolic profiling. (A) Heatmap of metabolic profiles at different time points after cobra venom intra-ION injection. (B) Heatmap of metabolic profiles in different groups of rats. 
Table 2 Identified Metabolites from the Serum Collected from TN Rats Received Curcumin Treatment or Vehicle

\begin{tabular}{|c|c|c|c|c|c|c|c|c|}
\hline VIP & $M / Z$ & RT & Biomarker & $\begin{array}{l}{[M} \\
+X]^{+}\end{array}$ & $\begin{array}{l}\text { Adduct } \\
\text { MW }\end{array}$ & Delta & $\begin{array}{l}\text { Ratio (Sham/Cobra } \\
\text { Venom/Curcumin) }\end{array}$ & $\begin{array}{l}\text { Ratio }(1 / 2 / \\
4 / 6 \text { Weeks })\end{array}$ \\
\hline 2.11 & 400.3416 & 23.1 & Thyroxine & $\begin{array}{l}\mathrm{M}+\mathrm{H} \\
+\mathrm{Na}\end{array}$ & 400.341588 & 0.000012 & $1.23 / 0 / 1.00$ & $0 / 0 / 0 / 0$ \\
\hline 1.98 & 563.3063 & 20.51 & Tauroursodeoxycholic acid & $\begin{array}{l}\mathrm{M} \\
+\mathrm{ACN} \\
+\mathrm{Na}\end{array}$ & $563.3 \mid 2524$ & 0.006224 & $1.00 / 0 / 0$ & $0 / 1.00 / 0 / 0$ \\
\hline 1.96 & 352.3379 & 10.09 & $\mathrm{CE}(20: 0)$ & $\begin{array}{l}\mathrm{M}+\mathrm{H} \\
+\mathrm{Na}\end{array}$ & 352.321813 & 0.016087 & $1.00 / 6.02 / 0$ & $\begin{array}{l}1.70 / 1.37 / \\
1.19 / 1.00\end{array}$ \\
\hline 1.96 & $553.387 I$ & 30.44 & LysoPE(22:I(I3Z)/0:0) & $\mathrm{M}+\mathrm{NH} 4$ & $553.3976 \mid 3$ & 0.010513 & $9.74 / 1.00 / 11.44$ & $0 / 0 / 0 / 1.00$ \\
\hline 1.9 & 834.8802 & 1.14 & Somatostatin & $\begin{array}{l}\mathrm{M}+\mathrm{H} \\
+\mathrm{NH} 4\end{array}$ & 834.886702 & 0.006502 & $12.02 / 1.00 / 0$ & $\begin{array}{l}2.51 / 3.82 / \\
3.89 / 1.00\end{array}$ \\
\hline 1.82 & 810.5966 & 33.55 & $P C(20: I(I I Z) / / 6: 0)$ & $\mathrm{M}+\mathrm{Na}$ & 810.598323 & 0.001723 & $12.55 / 1.00 / 5.54$ & $\begin{array}{l}3.81 / / 2.28 / \\
7.64 / 1.00\end{array}$ \\
\hline 1.77 & $1,107.617$ & 20.49 & Ganglioside GTIc (dI8:0/24:0) & $\mathrm{M}+2 \mathrm{H}$ & I, I07.598I7I & 0.018829 & $1.00 / 0 / 0$ & $\begin{array}{l}\mathrm{I} .07 / 1.45 / \\
\mathrm{I} .00 / 0\end{array}$ \\
\hline 1.7 & $1,531.913$ & 21.79 & Ganglioside GM2 (dI8:I/23:0) & $\begin{array}{l}\mathrm{M} \\
+\mathrm{DMSO} \\
+\mathrm{H}\end{array}$ & I,531.928052 & 0.014852 & I4.53/I5.38/I & $\begin{array}{l}1.00 / 1.34 / \\
1.52 / 1.49\end{array}$ \\
\hline 1.7 & $1,088.6519$ & 22.39 & $\begin{array}{l}\text { Trihexosylceramide (dI8:I/9Z- } \\
\mid 8: 1)\end{array}$ & $M+K$ & $\mathrm{I}, 088.649373$ & 0.002527 & $1.65 / 1.00 / 0$ & $\begin{array}{l}1.05 / 1.61 / \\
1.74 / 1.00\end{array}$ \\
\hline 1.69 & $1,132.6215$ & 20.85 & Ganglioside GTIb (dI8:0/26:0) & $\begin{array}{l}\mathrm{M}+\mathrm{H} \\
+\mathrm{Na}\end{array}$ & I, I 32.604792 & 0.016708 & $1.47 / 1.00 / 1.04$ & $\begin{array}{l}1.04 / 1.49 / \\
1.05 / 1.00\end{array}$ \\
\hline 1.69 & 539.3086 & 20.44 & I-Oleoylglycerophosphoserine & $\mathrm{M}+\mathrm{NH} 4$ & 539.309192 & 0.000592 & $2.47 / 1.00 / 0$ & $\begin{array}{l}1.00 / 1.95 / \\
2.16 / 1.09\end{array}$ \\
\hline 1.68 & 264.2662 & 19.3 & Cer(dI8:I/I4:0) & $\begin{array}{l}\mathrm{M}+\mathrm{H} \\
+\mathrm{NH} 4\end{array}$ & 264.260947 & 0.005253 & $7.59 / 7.81 / 0$ & $\begin{array}{l}1.00 / 1.31 / \\
1.87 / 2.01\end{array}$ \\
\hline 1.68 & 572.368 & 22.71 & $P C(I 8: I(9 Z) e / 2: 0)$ & $\mathrm{M}+\mathrm{Na}$ & 572.368658 & 0.000658 & $5.71 / 1.00 / 1.43$ & $\begin{array}{l}4.04 / 4.14 / \\
3.49 / 1.00\end{array}$ \\
\hline 1.64 & 504.304 & 20.6 & LysoPE(0:0//8:0) & $\mathrm{M}+\mathrm{Na}$ & 504.306057 & 0.002057 & $1.29 / 1.00 / 0$ & $\begin{array}{l}3.58 / 1.00 / \\
1.38 / 3.92\end{array}$ \\
\hline 1.64 & 542.3227 & 19.73 & $\begin{array}{l}\text { LysoPC }(20: 5 \\
(5 Z, 8 Z,|I Z,| 4 Z, 17 Z))\end{array}$ & $\mathrm{M}+\mathrm{H}$ & $542.324 I 15$ & 0.001415 & $9.73 / 5.47 / 1.00$ & $\begin{array}{l}1.20 / 1.65 / \\
1.79 / 1.00\end{array}$ \\
\hline 1.6 & $\mathrm{I}, 530.9088$ & 21.79 & Ganglioside GD3 (dI8:0/22:0) & $\mathrm{M}+\mathrm{H}$ & $\mathrm{I}, 530.92540 \mathrm{I}$ & 0.016601 & $4.86 / 5.22 / 1.00$ & $\begin{array}{l}1.00 / 1.34 / \\
1.52 / 1.49\end{array}$ \\
\hline 1.56 & 520.3376 & 20.84 & LysoPC $(18: 2(9 Z, \mid 2 Z))$ & $\mathrm{M}+\mathrm{H}$ & 520.339765 & 0.002165 & $1.4 \mathrm{I} / \mathrm{I} .4 \mathrm{I} / \mathrm{I} .00$ & $\begin{array}{l}1.12 / 1.00 / \\
1.13 / 1.06\end{array}$ \\
\hline 1.56 & 540.3058 & 21.79 & LysoPC(I8:3(9Z,I2Z,I5Z)) & $\mathrm{M}+\mathrm{Na}$ & 540.306057 & 0.000257 & I.34/1.33/1.00 & $\begin{array}{l}1.00 / 1.20 / \\
1.28 / 1.27\end{array}$ \\
\hline 1.56 & 506.299 & 22.4 & Lithocholyltaurine & $\mathrm{M}+\mathrm{Na}$ & 506.291062 & 0.007938 & $1.91 / 1.60 / 1.00$ & $\begin{array}{l}1.13 / 1.17 / \\
1.25 / 1.00\end{array}$ \\
\hline
\end{tabular}

(Continued) 
Table 2 (Continued).

\begin{tabular}{|c|c|c|c|c|c|c|c|c|}
\hline VIP & $M / Z$ & RT & Biomarker & $\begin{array}{l}{[\mathrm{M}} \\
+\mathrm{X}]^{+}\end{array}$ & $\begin{array}{l}\text { Adduct } \\
\text { MW }\end{array}$ & Delta & $\begin{array}{l}\text { Ratio (Sham/Cobra } \\
\text { Venom/Curcumin) }\end{array}$ & $\begin{array}{l}\text { Ratio }(1 / 2 / \\
4 / 6 \text { Weeks) }\end{array}$ \\
\hline 1.55 & 566.3179 & 20.96 & LysoPC(20:4(8Z, I IZ, I4Z, I7Z)) & $\mathrm{M}+\mathrm{Na}$ & 566.321707 & 0.003807 & $1.14 / 1.00 / 1.41$ & $\begin{array}{l}1.51 / 1.17 / \\
1.00 / 1.09\end{array}$ \\
\hline 1.55 & 541.3245 & 22.4 & I-Stearoylglycerophosphoserine & $\mathrm{M}+\mathrm{NH} 4$ & 541.324842 & 0.000342 & $2.59 / 1.95 / 1.00$ & $\begin{array}{l}1.00 / 1.88 / \\
2.03 / 1.55\end{array}$ \\
\hline 1.54 & 569.3402 & 20.74 & Deoxycholic acid 3-glucuronide & $\mathrm{M}+\mathrm{H}$ & 569.332024 & 0.008176 & $1.61 / 1.22 / 1.00$ & $\begin{array}{l}1.00 / 1.43 / \\
1.11 / 1.03\end{array}$ \\
\hline 1.53 & 510.3868 & 25.74 & LysoPC(O-|8:0) & $\mathrm{M}+\mathrm{H}$ & 510.391801 & 0.005001 & $0 / 3.00 / 1.00$ & $\begin{array}{l}1.00 / 0 / 3.61 / \\
6.27\end{array}$ \\
\hline 1.53 & 413.2673 & 23.63 & 9'-Carboxy-alpha-chromanol & $\mathrm{M}+\mathrm{Na}$ & 413.266228 & 0.001072 & $0 / 3.54 / 1.00$ & $\begin{array}{l}2.84 / 1.00 / \\
1.34 / 6.01\end{array}$ \\
\hline 1.53 & 522.3531 & 22.41 & LysoPC(I8: I(9Z)) & $\mathrm{M}+\mathrm{H}$ & 522.355416 & 0.002316 & $1.61 / 1.42 / 1.00$ & $\begin{array}{l}1.20 / 1.16 / \\
1.20 / 1.00\end{array}$ \\
\hline 1.51 & 526.2891 & 20.59 & LysoPE(0:0/20:3(8Z, I IZ, I4Z)) & $\mathrm{M}+\mathrm{Na}$ & 526.290407 & 0.001307 & $2.85 / 2.92 / 1.00$ & $\begin{array}{l}1.00 / 1.39 / \\
1.39 / 1.37\end{array}$ \\
\hline 1.51 & 466.3025 & 22.42 & LysoPC(I4:I(9Z)) & $\mathrm{M}+\mathrm{H}$ & 466.292815 & 0.009685 & $2.56 / 7.63 / 1.00$ & $\begin{array}{l}1.00 / 1.15 / \\
6.69 / 8.95\end{array}$ \\
\hline 1.5 & 494.3218 & 20.09 & LysoPC(16:I(9Z)) & $\mathrm{M}+\mathrm{H}$ & 494.324115 & 0.002315 & $3.10 / 1.70 / 1.00$ & $\begin{array}{l}1.53 / 2.09 / \\
1.66 / 1.00\end{array}$ \\
\hline 1.5 & 546.3526 & 21.68 & LysoPC(18:0) & $\mathrm{M}+\mathrm{Na}$ & 546.353008 & 0.000408 & $2.34 / 1.22 / 1.00$ & $\begin{array}{l}2.15 / 2.62 / \\
1.77 / 1.00\end{array}$ \\
\hline
\end{tabular}

potential roles in the development of chronic pain and chronic pain induced depression (Figure 6).

\section{Discussion}

In this study, we investigate the metabolic mechanism underlying chronic pain-induced depression through using a previously described trigeminal neuralgia rat model. Significant depression-like behaviors indicated by decreased sucrose preference and prolonged immobility during swimming, were observed in rats with trigeminal neuralgia. Furthermore, curcumin treatment may alleviate mechanical allodynia and depression-like behaviors in rats with trigeminal neuralgia through regulating metabolites and metabolic pathways.

Previous studies on the mechanisms of depression induced by chronic pain were mainly focused on psychology, anatomy, and neurochemistry. It is still unclear if the changes of serum metabolites under neuropathy conditions contribute to chronic pain and its induced depression. As sensitive markers of physiological activity, small molecule metabolites are the final products of cellular activities, which may reflect the abnormal molecular changes contributing to pain and pain-induced depression. In our study, we discovered 29 metabolite biomarkers in the blood serum from TN rats, which involve in phospholipids, lipids, cholic acids, and ganglioside metabolisms, etc (Figure 7).

Abundant studies have reported that neurotransmitters were changed by chronic pain in depression patients. ${ }^{26,27}$ In the present study, through metabolic profiling we found some hormones such as thyroxine and somatostatin were downregulated in $\mathrm{TN}$ rats. Both of them can regulate the endocrine system as neurotransmitters. ${ }^{28}$ Previous studies have indicated that thyroid dysfunction was related to depression in young patients. In animal model studies, rats with subclinical hypothyroidism showed depression-like behaviors indicated by increased immobility in both forced swimming and tail suspension tests. ${ }^{29-31}$ Somatostatin receptor subtype 4 was also found to be involved in anxiety and depression-like behaviors in a mouse model. ${ }^{32}$ 


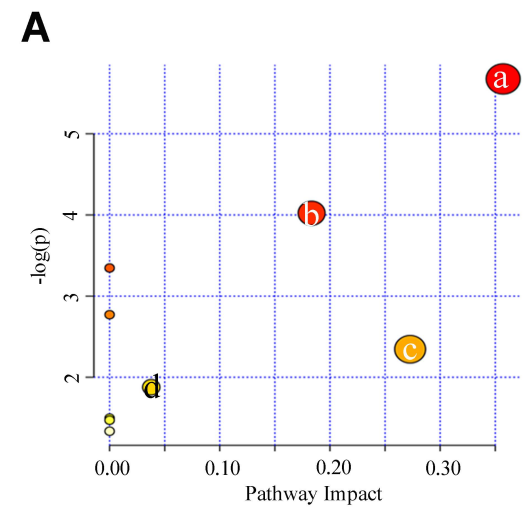

D

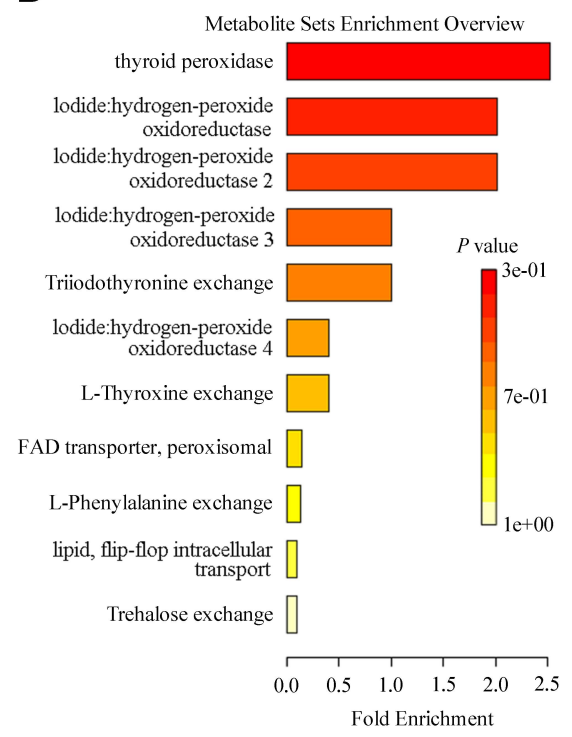

B

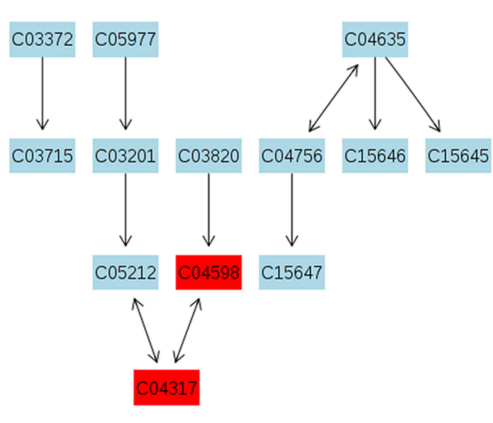

$E$
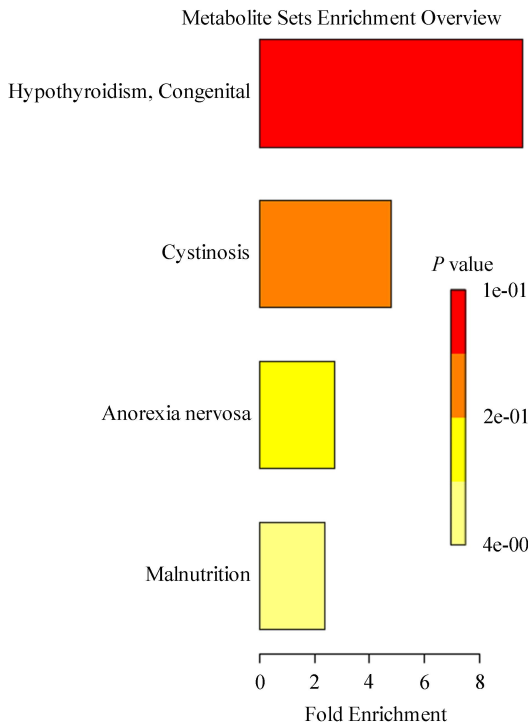

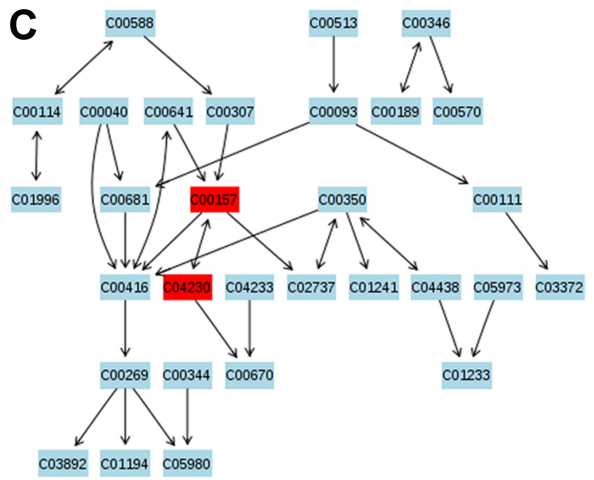

$\mathbf{F}$
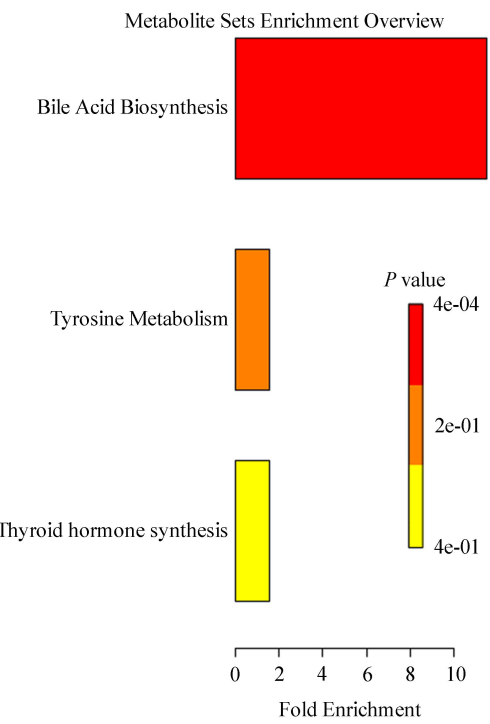

Figure 6 Pathway enrichment analysis of metabolites changed by curcumin treatment. (A) Pathway analysis of the effects of curcumin treatment on (a) Ether lipid metabolism, (b) Glycerophospholipid metabolism, (c) Pentose and glucuronate interconversions, and (d) Starch and sucrose metabolism in TN rats. (B) Glycerophospholipid metabolism (C04598: 2-Acetyl-I-alkyl-sn-glycero-3-phosphocholine, C043I7: I-Alkyl-2-lyso-sn-glycero-3-phosphocholine). (C) Ether lipid metabolism (C00I57: Phosphatidylcholine, C04230: I-Acyl-sn-glycero-3-phosphocholine. C03033: Glucuronide). (D-F) The enrichment pathway analysis of the effects of curcumin treatment in $\mathrm{TN}$ rats.

We also detected a series of abnormal changes in phospholipid metabolism in TN rats, such as progressively decreased production of $\mathrm{PC}(18: 1(9 \mathrm{Z}) \mathrm{e} / 2: 0))$ and LysoPC (20:4(8Z,11Z,14Z,17Z), and increased production of LysoPC(O-18:0) and LysoPC(14:1(9Z)). Phospholipids, ingredients for biological membranes, bile, and surface active substances participated in the recognition and signal transduction performed by cell membrane proteins. ${ }^{33,34} \mathrm{PC}$ is a precursor of acetylcholine, which is a neurotransmitter associated with memory and cognition, ${ }^{35}$ and can be transformed from PS or PE. PC belonging to (20:1(11Z)/16:0) phosphatidylcholine (KEGG Database Compound ID C00157) and lysoPC (20:5(5Z,8Z,11Z,14Z,17Z)), lysoPC (18:2(9Z,12Z)), lysoPC (18:3(9Z,12Z,15Z)), lysoPC (20:4 (8Z,11Z,14Z,17Z)), lysoPC (18:1(9Z)), lysoPC (14:1(9Z)),
lysoPC (16:1(9Z)), and lysoPC (18:0) belonging to 2-Lysolecithin (KEGG Database Compound ID C04230) are two big groups of glycerophospholipid and important for various biological functions. Under TN condition, we found phosphatidylcholine was progressively decreased; and on the other hand, 2-Lysolecithin was increased suggesting their potential roles in the development of chronic pain and the pain related depression.

The altered ganglioside metabolism found in our TN models also confirmed its important role in depression reported by a previous study. ${ }^{36-38}$

It has been widely believed that there is a close correlation between depression and liver diseases. A recent study found lipid metabolism in liver was significantly changed in a mouse model of depression, ${ }^{39}$ demonstrated by increased 


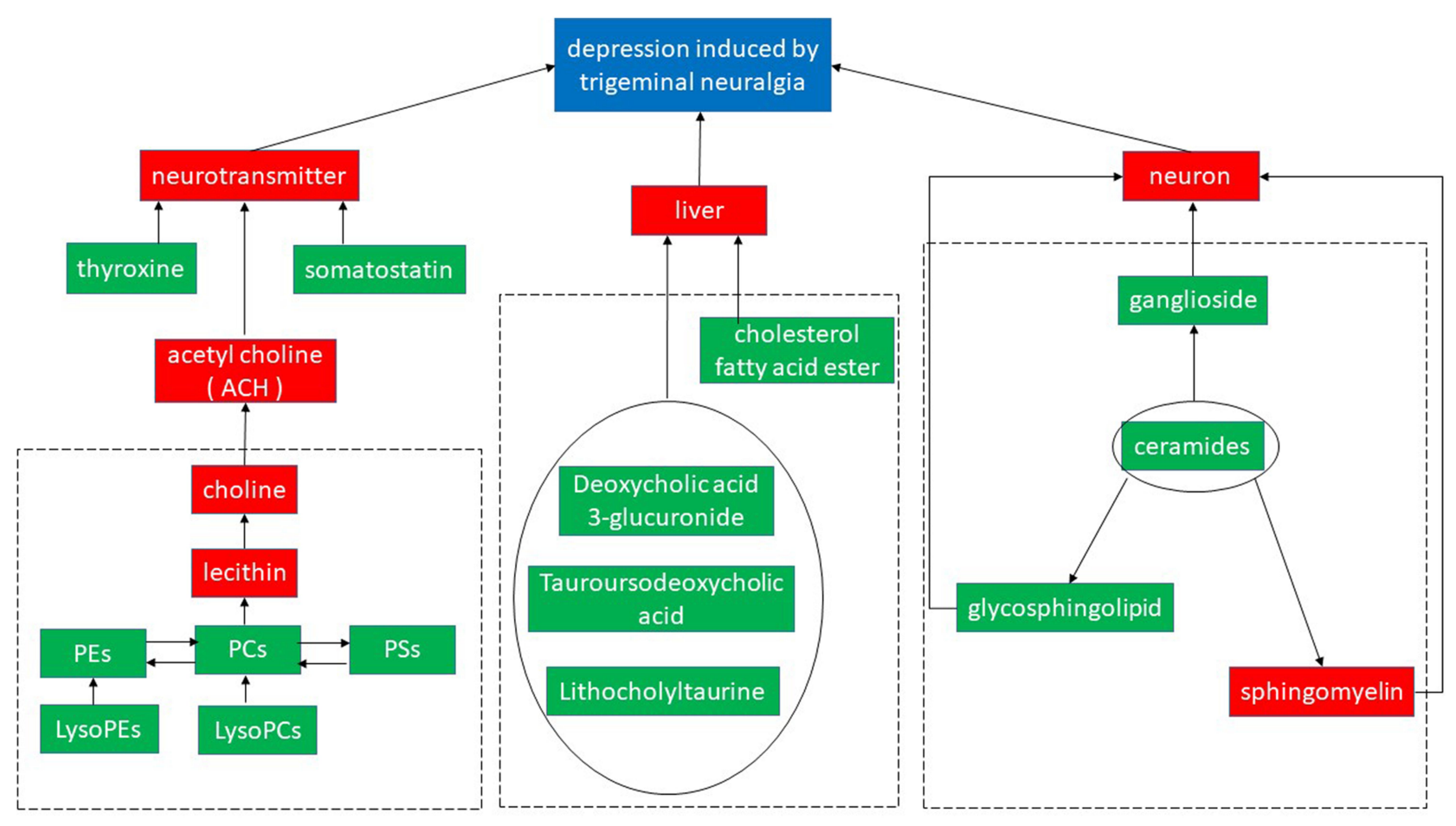

Figure 7 The effects of curcumin treatment on the expression of serum metabolite in TN rats. Differentially expressed metabolite pathways and their associated signaling networks (potential biomarkers were labeled by green solid box.).

liver secreting lecithin cholesterol acyltransferase, which enhanced the blood level of lysophosphatidyl choline. Consistent with these findings, we also detected metabolites changes related to bile acid metabolism, such as CE (20:0), tauroursodeoxycholic acid, ${ }^{40}$ lithocholyltaurine, ${ }^{41}$ and deoxycholic acid 3 -glucuronide. ${ }^{42}$

The benefits to the nervous system from curcumin have been intensively studied. In summary, curcumin can modulate the expression of monoamine oxidase, serotonin, dopamine, and neurotropic factors, and regulate the hypothalamus pituitary adrenal axis, and hippocampal neurogenesis and neuroplasticity, which are involved in the development of depression induced by chronic pain. ${ }^{43-47}$ Antinociceptive and antidepressant properties of curcumin have been evidenced in quite a few of the studies done in animal models. ${ }^{48-51}$ Moreover, curcumin was suggested to improve depressive symptoms in several clinical trials. ${ }^{49,52}$ A recent meta-analysis also suggested that curcumin as an add-on therapy may improve depressive and anxiety symptoms in human patients. ${ }^{53}$

In the present study, consistent with previous studies, we found curcumin effectively relieve mechanical allodynia and pain related depression in our TN rat models. More importantly, our results demonstrated that curcumin treatment normalized two most significant glycerophospholipid metabolism changes in $\mathrm{TN}$ rats by increasing the expression of $\mathrm{PC}(18: 1(9 \mathrm{Z}) \mathrm{e} / 2: 0)$ and $\operatorname{LysoPC}(20: 4$ $(8 Z, 11 Z, 14 Z, 17 Z))$ and decreasing the expression of LysoPC(O-18:0) and LysoPC(14:1(9Z)). Normalizing dysregulated glycerophospholipid metabolism under neuropathic condition may be a newly discovered mechanism, through which curcumin reduces chronic pain and improves pain related depression symptoms.

We admit that there are some limitations in this study. Based on our results we cannot exclude the possibility that our findings on the changes in serum metabolomic in neuropathic condition was the result instead of reasons of chronic pain, which had been found altering sphingomyelin-ceramide metabolism in the dorsal horn in a neuropathic pain model. ${ }^{54}$ Furthermore, the corresponding positive drug should be done as the control in animal experiments. In future studies, to avoid adaptation, appropriate control groups of animals would be added to forced swim test, if it has to be performed in multiple time points.

\section{Conclusion}

In this study, we found that metabolisms of ether lipid and glycerophospholipid were significantly changed in 
trigeminal nerve neuropathy condition, and may contribute to the generation of chronic pain and the correlated depression symptoms. Curcumin could alleviate pain and depression by modulating small molecule metabolite changes. The roles of small molecule metabolite changes in chronic pain conditions has only been studied in recent years, and has been proved to be a very promising research direction.

\section{Abbreviations}

ION, infraorbital nerve; MWT, mechanical withdrawal thresholds; HPLC, high performance liquid chromatography; SD, Sprague Dawley; LCMS-IT-TOF, liquid chromatography liquid chromatography-ion trap-time of flight; PCA, principal component analysis; PLS-DA, partial least squares discriminant analysis. $\mathrm{TN}$, trigeminal neuropathy.

\section{Data Sharing Statement}

There is no data, software, databases, and application/tool available apart from those reported in the present study. All data is provided in the manuscript and Supplementary $\underline{\text { Table } 1}$ and Supplementary Figures 1 and 2 . Data sharing statements: Participants gave informed consent for anonymized data sharing. The full data set is available on reasonable request from the corresponding author, Dr Zhang zhangli8828@163.com.

\section{Details of Ethical Approval}

All animal procedures performed in this study were reviewed and approved by the Institutional Animal Care and Use Committee in Beijing Friendship Hospital, Capital Medical University (Beijing, China) and were conducted in accordance with the guidelines of the International Association for the Study of Pain.

\section{Consent for Publication}

Not applicable.

\section{Acknowledgments}

This work was supported by the National Natural Science Foundation of China (81901125) and the Research Foundation of Beijing Friendship Hospital, Capital Medical University \# yyqdkt2018-23 (LX.A.). The authors thank Fan Liu for discussions on data analysis and manuscript drafting.

\section{Author Contributions}

All authors made substantial contributions to conception and design, acquisition of data, or analysis and interpretation of data; took part in drafting the article or revising it critically for important intellectual content; agreed to submit to the current journal; gave final approval of the version to be published; and agree to be accountable for all aspects of the work.

\section{Disclosure}

The authors declare that they have no competing interests.

\section{References}

1. Zhang M, Liu Y, Zhao M, et al. Depression and anxiety behaviour in a rat model of chronic migraine. $J$ Headache Pain. 2017;18(1):27. doi:10.1186/s10194-017-0736-z

2. Shi M, Wang JY, Luo F. Depression shows divergent effects on evoked and spontaneous pain behaviors in rats. $j$ Pain. 2010;11 (3):219-229. doi:10.1016/j.jpain.2009.07.002

3. Baune BT, Miller R, McAfoose J, Johnson M, Quirk F, Mitchell D. The role of cognitive impairment in general functioning in major depression. Psychiatry Res. 2010;176(2-3):183-189. doi:10.1016/j. psychres.2008.12.001

4. Felix RH, Almeida CB, Cremaschi RC, Coelho FM, Santos FC. Paininduced depression is related to overestimation of sleep quality in a very elderly population with pain. Arq Neuropsiquiatr. 2017;75 (1):25-29. doi:10.1590/0004-282x20160186

5. Apkarian AV, Sosa Y, Sonty S, et al. Chronic back pain is associated with decreased prefrontal and thalamic gray matter density. J Neurosci. 2004;24(46):10410-10415. doi:10.1523/JNEUROSCI.2541-04.2004

6. Grachev ID, Fredrickson BE, Apkarian AV. Abnormal brain chemistry in chronic back pain: an in vivo proton magnetic resonance spectroscopy study. Pain. 2000;89(1):7-18. doi:10.1016/S0304-3959(00)00340-7

7. Egloff N, Wegmann B, Juon B, Stauber S, von Kanel R, Vogelin E. The impact of anxiety and depressive symptoms on chronic pain in conservatively and operatively treated hand surgery patients. $J$ Pain Res. 2017;10:259-263. doi:10.2147/JPR.S116674

8. O'Gorman A, Brennan L. The role of metabolomics in determination of new dietary biomarkers. Proc Nutr Soc. 2017;76(3):295-302. doi:10.1017/S0029665116002974

9. Allard PM, Genta-Jouve G, Wolfender JL. Deep metabolome annotation in natural products research: towards a virtuous cycle in metabolite identification. Curr Opin Chem Biol. 2017;36:40-49. doi:10.1016/j.cbpa.2016.12.022

10. Lestari ML, Indrayanto G. Curcumin. Profiles Drug Subst Excip Relat Methodol. 2014;39:113-204.

11. Mahady GB, Pendland SL, Yun G, Lu ZZ. Turmeric (Curcuma longa) and curcumin inhibit the growth of Helicobacter pylori, a group 1 carcinogen. Anticancer Res. 2002;22(6c):4179-4181.

12. Reddy RC, Vatsala PG, Keshamouni VG, Padmanaban G, Rangarajan PN. Curcumin for malaria therapy. Biochem Biophys Res Commun. 2005;326(2):472-474. doi:10.1016/j.bbrc.2004.11.051

13. Vera-Ramirez L, Pérez-Lopez P, Varela-Lopez A, Ramirez-Tortosa M, Battino M, Quiles JL. Curcumin and liver disease. Biofactors. 2013;39(1):88-100. doi:10.1002/biof.1057

14. Wright LE, Frye JB, Gorti B, Timmermann BN, Funk JL. Bioactivity of turmeric-derived curcuminoids and related metabolites in breast cancer. Curr Pharm Des. 2013;19(34):6218-6225. doi:10.2174/ 1381612811319340013

15. Gupta SC, Patchva S, Aggarwal BB. Therapeutic roles of curcumin: lessons learned from clinical trials. AAPS J. 2013;15(1):195-218. doi:10.1208/s12248-012-9432-8

16. Kulkarni SK, Bhutani MK, Bishnoi M. Antidepressant activity of curcumin: involvement of serotonin and dopamine system. Psychopharmacology. 2008;201(3):435-442. doi:10.1007/s00213008-1300-y 
17. Wang R, Xu Y, Wu HL, et al. The antidepressant effects of curcumin in the forced swimming test involve 5-HT1 and 5-HT2 receptors. Eur J Pharmacol. 2008;578(1):43-50. doi:10.1016/j.ejphar.2007.08.045

18. Lin TY, Lu CW, Wang CC, Wang YC, Wang SJ. Curcumin inhibits glutamate release in nerve terminals from rat prefrontal cortex: possible relevance to its antidepressant mechanism. Prog Neuropsychopharmacol Biol Psychiatry. 2011;35(7):1785-1793. doi:10.1016/j.pnpbp.2011.06.012

19. Zhang $\mathrm{L}, \mathrm{Xu} \mathrm{T}$, Wang $\mathrm{S}$, et al. Curcumin produces antidepressant effects via activating MAPK/ERK-dependent brain-derived neurotrophic factor expression in the amygdala of mice. Behav Brain Res. 2012;235(1):67-72. doi:10.1016/j.bbr.2012.07.019

20. An JX, He Y, Qian XY, et al. A new animal model of trigeminal neuralgia produced by administration of cobra venom to the infraorbital nerve in the rat. Anesth Analg. 2011;113(3):652-656. doi:10.1213/ANE.0b013e3182245add

21. Zhao X, Wang C, Zhang JF, et al. Chronic curcumin treatment normalizes depression-like behaviors in mice with mononeuropathy: involvement of supraspinal serotonergic system and GABAA receptor. Psychopharmacology. 2014;231(10):2171-2187. doi:10.1007/s00213-013-3368-2

22. Wu Z, Qian XY, An JX, et al. Trigeminal neuralgia induced by cobra venom in the rat leads to deficits in abilities of spatial learning and memory. Pain Phys. 2015;18(2):E207-216.

23. Zhang L, Ding X, Wu Z, Wang M, Tian M. Curcumin alleviates pain and improves cognitive impairment in a rat model of cobra venom-induced trigeminal neuralgia. $J$ Pain Res. 2018;11:1095-1104. doi:10.2147/JPR.S162668

24. Wang J, Goffer Y, Xu D, et al. A single subanesthetic dose of ketamine relieves depression-like behaviors induced by neuropathic pain in rats. Anesthesiology. 2011;115(4):812-821. doi:10.1097/ ALN.0b013e31822f16ae

25. Ma X, Chi YH, Niu M, et al. Metabolomics coupled with multivariate data and pathway analysis on potential biomarkers in cholestasis and intervention effect of paeonia lactiflora pall. Front Pharmacol. 2016;7:14. doi:10.3389/fphar.2016.00014

26. Goesling J, Clauw DJ, Hassett AL. Pain and depression: an integrative review of neurobiological and psychological factors. Curr Psychiatry Rep. 2013;15(12):421. doi:10.1007/s11920-013-0421-0

27. Singh L, Kaur A, Garg S, Bhatti R. Skimmetin/osthole mitigates pain-depression dyad via inhibiting inflammatory and oxidative stress-mediated neurotransmitter dysregulation. Metab Brain Dis. 2020. doi:10.1007/s11011-020-00604-4

28. Arroba AI, Mazzeo A, Cazzoni D, et al. Somatostatin protects photoreceptor cells against high glucose-induced apoptosis. Mol Vis. 2016;22:1522-1531.

29. Capo-chichi CD, Gueant JL, Lefebvre E, et al. Riboflavin and riboflavin-derived cofactors in adolescent girls with anorexia nervosa. Am J Clin Nutr. 1999;69(4):672-678. doi:10.1093/ajen/69.4.672

30. Sjoberg S, Eriksson M, Nordin C. L-thyroxine treatment and neurotransmitter levels in the cerebrospinal fluid of hypothyroid patients: a pilot study. Eur j Endocrinol. 1998;139(5):493-497. doi:10.1530/ eje. 0.1390493

31. Chandurkar V, Shik J, Randell E. Exacerbation of underlying hypothyroidism caused by proteinuria and induction of urinary thyroxine loss: case report and subsequent investigation. Endocrine Pract. 2008;14(1):97-103. doi:10.4158/EP.14.1.97

32. Scheich B, Gaszner B, Kormos V, et al. Somatostatin receptor subtype 4 activation is involved in anxiety and depression-like behavior in mouse models. Neuropharmacology. 2016;101:204-215. doi:10.1016/j.neuropharm.2015.09.021

33. Dang VT, Huang A, Zhong LH, Shi Y, Werstuck GH. Comprehensive plasma metabolomic analyses of atherosclerotic progression reveal alterations in glycerophospholipid and sphingolipid metabolism in apolipoprotein E-deficient Mice. Sci Rep. 2016;6:35037. doi:10.1038/srep35037
34. Romay MC, Che N, Becker SN, et al. Regulation of NF-kappaB signaling by oxidized glycerophospholipid and IL-1beta induced miRs-21-3p and $-27 \mathrm{a}-5 \mathrm{p}$ in human aortic endothelial cells. $J$ Lipid Res. 2015;56(1):38-50. doi:10.1194/jlr.M052670

35. Liu XJ, Zhou YZ, Li ZF, et al. Anti-depressant effects of Xiaoyaosan on rat model of chronic unpredictable mild stress: a plasma metabonomics study based on NMR spectroscopy. J Pharm Pharmacol. 2012;64(4):578-588. doi:10.1111/j.2042-7158.2011.01412.x

36. Małecki A, Małecka E, Trzeciak HI. [Gangliosides in neurological pharmacotherapy]. Neurol Neurochir Pol. 1992;26(5):695-701. [Article in Polish].

37. Gronnier J, Germain V, Gouguet P, Cacas JL, Mongrand S. GIPC: glycosyl Inositol Phospho Ceramides, the major sphingolipids on earth. Plant Signal Behav. 2016;11(4):e1152438. doi:10.1080/ 15592324.2016.1152438

38. Jiang B, Song L, Wang CN, Zhang W, Huang C, Tong LJ. Antidepressant-like effects of GM1 ganglioside involving the BDNF signaling cascade in mice. Int $j$ Neuropsychopharmacol. 2016;19:9. doi:10.1093/ijnp/pyw046

39. Wu Y, Tang J, Zhou C, et al. Quantitative proteomics analysis of the liver reveals immune regulation and lipid metabolism dysregulation in a mouse model of depression. Behav Brain Res. 2016;311:330-339. doi:10.1016/j.bbr.2016.05.057

40. Rolo AP, Palmeira CM, Holy JM, Wallace KB. Role of mitochondrial dysfunction in combined bile acid-induced cytotoxicity: the switch between apoptosis and necrosis. Toxicol Sci. 2004;79(1):196-204. doi: $10.1093 /$ toxsci/kfh078

41. Watanabe N, Kagawa T, Kojima S, et al. Taurolithocholate impairs bile canalicular motility and canalicular bile secretion in isolated rat hepatocyte couplets. World j Gastroenterol. 2006;12(33):5320-5325. doi:10.3748/wjg.v12.i33.5320

42. Goto J. [Chromatographic determination of bile acids in biological fluids with sensitive and selective detection]. Yakugaku Zassh. 1990;110(11):807-821. [Article in Japanese]. doi:10.1248/ yakushi1947.110.11_807

43. Khatri DK, Juvekar AR. Kinetics of inhibition of monoamine oxidase using curcumin and ellagic acid. Pharmacogn Mag. 2016;12(Suppl 2):S116-120. doi:10.4103/0973-1296.182168

44. Kulkarni SK, Dhir A. An overview of curcumin in neurological disorders. Indian J Pharm Sci. 2010;72(2):149-154. doi:10.4103/ 0250-474X.65012

45. Chang XR, Wang L, Li J, Wu DS. Analysis of anti-depressant potential of curcumin against depression induced male albino wistar rats. Brain Res. 2016;1642:219-225. doi:10.1016/j.brainres.2016.03.010

46. Jangra A, Kwatra M, Singh T, et al. Piperine augments the protective effect of curcumin against lipopolysaccharide-induced neurobehavioral and neurochemical deficits in mice. Inflammation. 2016;39 (3):1025-1038. doi:10.1007/s10753-016-0332-4

47. Bortolato B, Miskowiak KW, Kohler CA, et al. Cognitive remission: a novel objective for the treatment of major depression? BMC Med. 2016;14:9. doi:10.1186/s12916-016-0560-3

48. Mittal N, Joshi R, Hota D, Chakrabarti A. Evaluation of antihyperalgesic effect of curcumin on formalin-induced orofacial pain in rat. Phytother Res. 2009;23(4):507-512. doi:10.1002/ptr.2662

49. Ng QX, Koh SSH, Chan HW, Ho CYX. Clinical use of curcumin in depression: a meta-analysis. J Am Med Dir Assoc. 2017;18 (6):503-508. doi:10.1016/j.jamda.2016.12.071

50. Choi GY, Kim HB, Hwang ES, et al. Curcumin alters neural plasticity and viability of intact hippocampal circuits and attenuates behavioral despair and COX-2 expression in chronically stressed rats. Mediators Inflamm. 2017;2017:6280925. doi: $10.1155 / 2017 / 6280925$

51. Kaufmann FN, Gazal M, Bastos CR, Kaster MP, Ghisleni G. Curcumin in depressive disorders: an overview of potential mechanisms, preclinical and clinical findings. Eur $J$ Pharmacol. 2016;784:192-198. doi:10.1016/j.ejphar.2016.05.026 
52. Al-Karawi D, Al Mamoori DA, Tayyar Y. The role of curcumin administration in patients with major depressive disorder: mini meta-analysis of clinical trials. Phytother Res. 2016;30(2):175-183. doi:10.1002/ptr.5524

53. Fusar-Poli L, Vozza L, Gabbiadini A, et al. Curcumin for depression: a meta-analysis. Crit Rev Food Sci Nutr. 2020;60(15):2643-2653. doi:10.1080/10408398.2019.1653260
54. Patti GJ, Yanes O, Shriver LP, et al. Metabolomics implicates altered sphingolipids in chronic pain of neuropathic origin. Nat Chem Biol. 2012;8(3):232-234. doi:10.1038/nchembio.767

\section{Publish your work in this journal}

The Journal of Pain Research is an international, peer reviewed, open access, online journal that welcomes laboratory and clinical findings in the fields of pain research and the prevention and management of pain. Original research, reviews, symposium reports, hypothesis formation and commentaries are all considered for publication. The manuscript management system is completely online and includes a very quick and fair peer-review system, which is all easy to use. Visit http:// www.dovepress.com/testimonials.php to read real quotes from published authors. 